\title{
“Titanium Oxide-Clay” as Adsorbent and Photocatalysts for Wastewater Treatment
}

\author{
Pohan Lemeyonouin Aliou Guillaume ${ }^{1^{*}}$, Andreea-Maria Chelaru ${ }^{2}$, Maria Visa ${ }^{2^{\star}}$ and Ouattara Lassiné ${ }^{3}$ \\ ${ }^{1}$ Université Péléforo Gon Coulibaly de Korhogo, UFR Sciences Biologiques, BP 1328 Korhogo, Côte d'lvoire \\ ${ }^{2}$ TransilvaniaUniversity of Brasov, RTD Dept. Renewable Energy Systems and Recycling, Romania, Eroilor 29, 500036 Brasov, Romania \\ ${ }^{3}$ Laboratoire de Chimie Physique, UFR SSMT, Université Félix Houphouët-Boigny de Cocody, Abidjan, 22 BP 582 Abidjan 22, Côte d'Ivoire
}

\begin{abstract}
A novel composite based on Titanium oxide and clays hydrothermally was synthesized to be used as substrate in advanced treatment of wastewaters. The treatment consists of one single step process combining photocatalysis and adsorption. The composite's crystalline structure is investigated by X-ray diffraction and FTIR, while atomic force microscopy (AFM) and scanning electron microscopy (SEM) are used to analyze the surface morphology. The adsorption capacity and photocatalytic properties of the material are tested on pollutants matrix containing dye (Methylene Blue) and heavy metal (cadmium cation). The results under optimized conditions indicate a good removal efficiency using this novel composite material.
\end{abstract}

Keywords: Cadmium cation; Methylene blue; Nanocomposite; Wastewater treatment

\section{Introduction}

Water, this essential element for life, is abundant on earth (estimated volume of about $\left.1.4 \times 109 \mathrm{~km}^{3}\right)$. However, $97.5 \%$ is salt water. Of the remaining $2.5 \%$ that is fresh water, $70 \%$ is frozen in the polar icecaps; the rest is mainly present as soil moisture or in inaccessible subterranean aquifers. Only less than $1 \%$ of the world's fresh water resources are readily available for human use; but this resource is very unevenly distributed [1]. Besides, the available fresh water is not always clean as it may contain natural toxic pollutants (heavy metals metal-complex dyes, bacteria). Excessive release of heavy metals into the environment due to industrialization and urbanization poses great problem worldwide. Metals such as cadmium, cooper, lead, are contained in wastewater derived from electroplating, mining, batteries, plastic and paint. Unlike organic pollutants, heavy metals cations do not degrade into harmless end products. Due to their high toxicity, they can cause many health disorders (to the central nervous system, kidneys and reproductive system) [2].

Besides, in many countries the significant numbers of textile industries are the main sources of fresh water pollution. The wastewaters from these industries are loaded with a considerable amount of dyes and surfactants. A variety of dyes are highly toxic for animals and humans, and affect water transparency reducing light penetration and gas solubility in water $[3,4]$, thus disturbing the structure of the ecosystem. These dyes are also highly soluble, and resistant to degradation by organisms. Consequently, their removal from wastewater remains a difficult, but necessary, task. Treatments such as chemical oxidation, precipitation and coagulation of the pollutants, reverse osmosis are less effective at commercial scale due to the high cost and complexity $[4,5]$ than adsorption process. Advanced Oxidation Processes (AOP) is modern chemical methods and effective for the treatment of water containing non-biodegradable/toxic substances and for the decontamination of drinking water [6-10].

Among the advanced oxidation processes (AOPs), photocatalysis is recognized for the ability to mineralize a wide range of organic compounds as it involves the generation of highly reactive radical species, predominantly the hydroxyl radical $(\mathrm{HO} \bullet)$ which is a powerful oxidative agent, active for degrading recalcitrant high molecules of dyes. In this purpose, many photocatalysts such as semiconductors:
$\mathrm{TiO}_{2}, \mathrm{SnO}_{2}, \mathrm{WO}_{3}[11]$, coupled semiconductors: $\mathrm{TiO}_{2} / \mathrm{SnO}_{2}, \mathrm{TiO}_{2} / \mathrm{ZnO}$ $\mathrm{TiO}_{2} / \mathrm{WO}_{3}[12]$ were reported.

Heterogeneous photocatalysis based on reactions onto the surface of wide band gap semiconductor $\left(\mathrm{TiO}_{2}\right)$ irradiated with solar or artificial light, are of interest because of their ability to mineralize organic pollutants. For simultaneous removal of heavy metals and dyes from the waste waters, several researchers have coated photocatalysts $\left(\mathrm{TiO}_{2}\right)$ onto a variety of surfaces like, modified fly ash [13], diatomite, bentonite and clay [14]. The $\mathrm{TiO}_{2}$ particles immobilized on adsorbent support can be more easily filtered, this is necessary for industrial applications. Thus, a new material, of zeolite-type, was developed in environmentally friendly conditions, as substrate in adsorption and as heterogeneous photocatalyst.

This paper presents the obtained results obtained the new synthetized composite (clay- $\mathrm{TiO}_{2}$ ) via mild hydrothermal synthesis, from Degussa P25 and clay of north of Ivory Coast in alkaline media, aimed to be used as substrate in the advanced treatment of wastewater loaded with dye (methylene blue) and heavy metal (cadmium).

\section{Experimental}

\section{Raw material}

The clay materials used in this work were collected from two different regions of north of Ivory Coast, namely: Katiola and Fronan.

${ }^{*}$ Corresponding authors: Pohan Lemeyonouin Aliou Guillaume, Universite Péléforo Gon Coulibaly de Korhogo, UFR Sciences Biologiques, BP 1328 Korhogo, Côte d'Ivoire, Tel: 2250755 32; E-mail: pohan.aliou@gmail.com

Maria Visa, TransilvaniaUniversity of Brasov, RTD Dept. Renewable Energy Systems and Recycling, Romania, Eroilor 29, Brasov, Romania, Tel: 400729109 355; E-mail: maria.visa@unitbv.ro

Received November 02, 2017; Accepted November 13, 2017; Published November 25, 2017

Citation: Guillaume PLA, Chelaru AM, Visa M, Lassiné O (2017) "Titanium OxideClay" as Adsorbent and Photocatalysts for Wastewater Treatment. J Membra Sci Technol 8: 176. doi:10.4172/2155-9589.1000176

Copyright: @ 2018 Guillaume PLA, et al. This is an open-access article distributed under the terms of the Creative Commons Attribution License, which permits unrestricted use, distribution, and reproduction in any medium, provided the original author and source are credited. 
The clay was named according to their color in French (Blanc (B), Vert $(\mathrm{V})$, and Rouge $(\mathrm{R})$ ).

\section{Reagents}

All the reagents were used as received without further treatment. The $\mathrm{TiO}_{2}$ used was from Degussa (Degussa P25, 80\% anatase and 20\% rutile; specific surface area $50 \mathrm{~m}^{2} \mathrm{~g}^{-1}$ and a mean particle size of $30 \mathrm{~nm}$ ), Cadmium chloride hemi(pentahydrate) $\left(\mathrm{CdCl}_{2} \cdot 2.5 \mathrm{H}_{2} \mathrm{O},<98 \%\right.$ purity) from ScharlauChemie S.A, methylene blue from Fluka $\left(\mathrm{C}_{16} \mathrm{H}_{18} \mathrm{~N}_{3} \mathrm{~S}\right)$, and sodium hydroxide $(\mathrm{NaOH}, \geq 99 \%)$ from Fulka.

\section{Material substrate preparation}

Before being used, the clay materials $(\mathrm{B}, \mathrm{V}$ and $\mathrm{R})$ were washed with ultra-pure water under mechanical stirring ( $100 \mathrm{rpm}$, Nahita GJ-1 stirrer) at room temperature $\left(22 \pm 1^{\circ} \mathrm{C}\right)$ for $24 \mathrm{~h}$, in order to remove the unwanted soluble compounds. The ratio between clay materials and ultra-pure water ratio was 1:10 (g: $\mathrm{mL})$. Afterwards the suspension was filtered and dried at $105-115^{\circ} \mathrm{C}$. The washed and dried clay materials were mechanically sieved (Analysette 3 Spartan) and the $40 \mu \mathrm{m}$ fraction was selected for experiments. During the hydrothermal process the washed clay materials was treated with $\mathrm{NaOH} 2 \mathrm{~N}$ solution. The modified clay materials were obtained under stirring in autoclave at $100^{\circ} \mathrm{C}$ and $5 \mathrm{~atm}$. After the reactions were completed, the suspended matter was washed with ultra-pure water until constant $\mathrm{pH}(\mathrm{pH}=9.9)$; afterwards it is filtered and dried at $105-115^{\circ} \mathrm{C}$ overnight. The modified clay materials obtained were denoted $\mathrm{BW}-\mathrm{NaOH}_{2 \mathrm{~N}}, \mathrm{VW}-\mathrm{NaOH}_{2 \mathrm{~N}}$ and $\mathrm{RW}-\mathrm{NaOH}_{2 \mathrm{~N}}$.

The composite substrate of clay and $\mathrm{TiO}_{2}\left(\mathrm{~B}-\mathrm{TiO}_{2}, \mathrm{~V}-\mathrm{TiO}\right.$ and $\mathrm{R}-\mathrm{TiO}_{2}$ ) was obtained mixing $30 \mathrm{~g}$ BW- $\mathrm{NaOH}_{2 \mathrm{~N}}$ (or $\mathrm{VW}-\mathrm{NaOH}_{2 \mathrm{~N}}$ or RW- $\mathrm{NaOH}_{2 \mathrm{~N}}$ ) with $30 \mathrm{~g}$ Degussa P25 with $36 \mathrm{~g} \mathrm{NaOH}$ (mass ratio 1:1:1.2). Hydrothermal synthesis parameters were: $\mathrm{T}=100^{\circ} \mathrm{C}, \mathrm{P}=5 \mathrm{~atm}$. during $24 \mathrm{~h}$.

\section{Characterization of the material substrate}

The crystalline structure of the composite substrate was investigated by XRD (Bruker D8 Discover Diffractometer), over the $2 \theta$ range 10 $70^{\circ}$. Morphology studies (roughness and macro pore size dis-tribution) were done using AFM (Ntegra Spectra, NT-MDT modelBL222RNTE); images were taken in semi-contact mode with golden silicon cantilever (NCSG10), with constant force $(0.15 \mathrm{~N} / \mathrm{m})$, having the tip radius of 10 $\mathrm{nm}$. Scanning was conducted on three or more different places with a certain area of $5 \times 5 \mu \mathrm{m}$ for each position, randomly chosen at a scanning grate of $1 \mathrm{~Hz}$. Further surface investigations were done using scanning electron microscopy (SEM, S-3400N-Hitachi) at an accelerating voltage of $20 \mathrm{KV}$. Surface composition was evaluated using energy dispersive $\mathrm{X}$-ray spectroscopy (EDS, Thermo Scientific Ultra Dry). Surface characterization was completed by microporosity analysis (AFM) and BET surface measurements (Autosorb-IQ-MP, Quantachrome Instruments). The information related to the functional groups on the surface was provided by FTIR (PerkinElmer BX II 75548).

\section{Adsorption and photocatalytic experiments}

Batch adsorption tests were done in open cylindrical flasks, at room temperature $\left(22 \pm 1^{\circ} \mathrm{C}\right)$ by mixing $0.1 \mathrm{~g}$ of substrate with $50 \mathrm{~mL}$ solution $\left(\mathrm{MB} ; \mathrm{MB}+\mathrm{Cd}^{2+}\right.$ ) under mechanical stirring. The adsorption duration of $\mathrm{MB}, \mathrm{Cd}^{2+}$ cations on the composite substrate $\left(\mathrm{B}-\mathrm{TiO}_{2}\right.$, $\mathrm{V}-\mathrm{TiO}_{2}$ and $\mathrm{R}-\mathrm{TiO}_{2}$ ): was varied up to $240 \mathrm{~min}$, followed by filtration and filtrate analysis. The initial concentrations of the pollutant systems were $0.01 \mathrm{~N} \mathrm{CdCl}_{2}$ prepared in bi-distilled water using $\mathrm{CdCl}_{2} \cdot 2.5 \mathrm{H}_{2} \mathrm{O}$ (ScharlauChemie S.A., <98\%purity) and $0.03125 \mathrm{mM}$, methylene blue $\left(\mathrm{C}_{16} \mathrm{H}_{18} \mathrm{~N}_{3} \mathrm{~S}\right.$; Fluka AG, reagent grade). The adsorption mechanism and the kinetic data were evaluated. These experiments are denoted with (A).
Photodegradation investigations were done on $\mathrm{B}-\mathrm{TiO}_{2}, \mathrm{~V}-\mathrm{TiO}_{2}$ and $\mathrm{R}-\mathrm{TiO}_{2}$ suspensions with the same composition as in the adsorption studies, with and without Fenton reactive and hydrogen peroxide (30\%), under UV irradiation, and the results are denoted with (F). The photocatalytic reactor equipped with three F18W/T8 black tubes (Philips), emitting UV-A light in the region of $340-400 \mathrm{~nm}$ and $\lambda_{\max }($ emission $)=365 \mathrm{~nm}$.

The mean value of the radiation flux intensity, reaching the middle of the reacting suspension was $3 \mathrm{Lx}$ (Mavolux5032B/USM) and the irradiance is $846 \mathrm{~W} \mathrm{~m}^{-2}$. During the adsorption studies the radiation flux intensity was in the range of $0.7-1.6 \mathrm{Lx}$, with an average irradiation of $215 \mathrm{~W} \mathrm{~m}^{-2}$.

During the kinetic studies, aliquots were taken at fixed moments (up to $360 \mathrm{~min}$ ) when stirring was briefly interrupted and, after filtration on $0.45 \mathrm{~mm}$ filter, the supernatant was analyses. Preliminary experiments proved that dyes losses due to adsorption on the beaker walls or on the filtering paper were negligible.

The initial and residual metal concentration in the aqueous solution was analyzed by AAS (Analytic Jena, ZEEnit 700, at $\lambda_{\mathrm{Cd}}=228.8 \mathrm{~nm}$ ) and the MB was analyzed by UV-vis spectrometry (Perkin Elmer Lambda 25 ), on the calibration curve registered at $\lambda=664 \mathrm{~nm}$, respectively.

The adsorption/photodegradation efficiency for the cadmium cations and dyes was evaluated using Equation (1):

$$
\eta=\frac{C_{0}-C_{t}}{C_{0}} \times 100
$$

Where: $c_{0}$ represents the initial concentration of the pollutants and $c_{t}$ represents the concentration of the pollutants at time $t$.

\section{Results and Discussion}

\section{The characterization of composites material}

The XRD analyses (Figure 1) show more complex composition for the new composites $\mathrm{B}-\mathrm{TiO}_{2}, \mathrm{~V}-\mathrm{TiO}_{2}$ and $\mathrm{R}-\mathrm{TiO}_{2}$, the structures are composed of the anatase phase mostly (Tables 1-3)

The crystalline structures of washed clay (B-W, V-W and R-W) and of the composites $\left(\mathrm{B}-\mathrm{TiO}_{2}, \mathrm{~V}-\mathrm{TiO}_{2}\right.$ and $\mathrm{R}-\mathrm{TiO}_{2}$ ) were comparatively investigated. The crystallite sizes were calculated using the Scherrer formula, Equation (2) [15].

$$
\tau=\frac{K \lambda}{\beta \cos \theta}
$$

Where: $\tau$ is the size of crystallites, $\mathrm{K}$ is the shape factor with a typical value $0.94, \lambda$ is the $\mathrm{X}$-ray wavelength (1.5406 $\AA$ ), $\beta$ is the line broadening at half the maximum intensity (of a peak), and $\theta$ is diffraction angle.

The XRD spectra (Figure 1) display overall crystalline percentages of $73.9 \%$ in $\mathrm{B}-\mathrm{W}, 82.1 \%$ in $\mathrm{V}-\mathrm{W}$ and $63.9 \%$ in R-W. The major crystalline components of washed clay are: $\mathrm{SiO}_{2}\left(\right.$ as $\alpha \mathrm{SiO}_{2}$ quartz, quartz syn, quartz low, cubic and orthorhombic $\mathrm{SiO}_{2}$ ). The XRD data show that the new substrates, $\mathrm{B}_{-} \mathrm{TiO}_{2}$ (for example), have well embedded the anatase phase (anatase syn with $237.7 \AA$ crystallite size) and the rutile - $\mathrm{TiO}_{2}$ (with $120.7 \AA$ crystallite size), one may conclude that the hydrothermal treatment supported a $\mathrm{TiO}_{2}$ recrystallization process on the microsized $\mathrm{B}-\mathrm{W}$, extending the crystallite dimensions. $\mathrm{B}-\mathrm{TiO}_{2}$ contains $\mathrm{TiO}_{2}$ polymorphs. New compounds are identified on the XRD spectra 


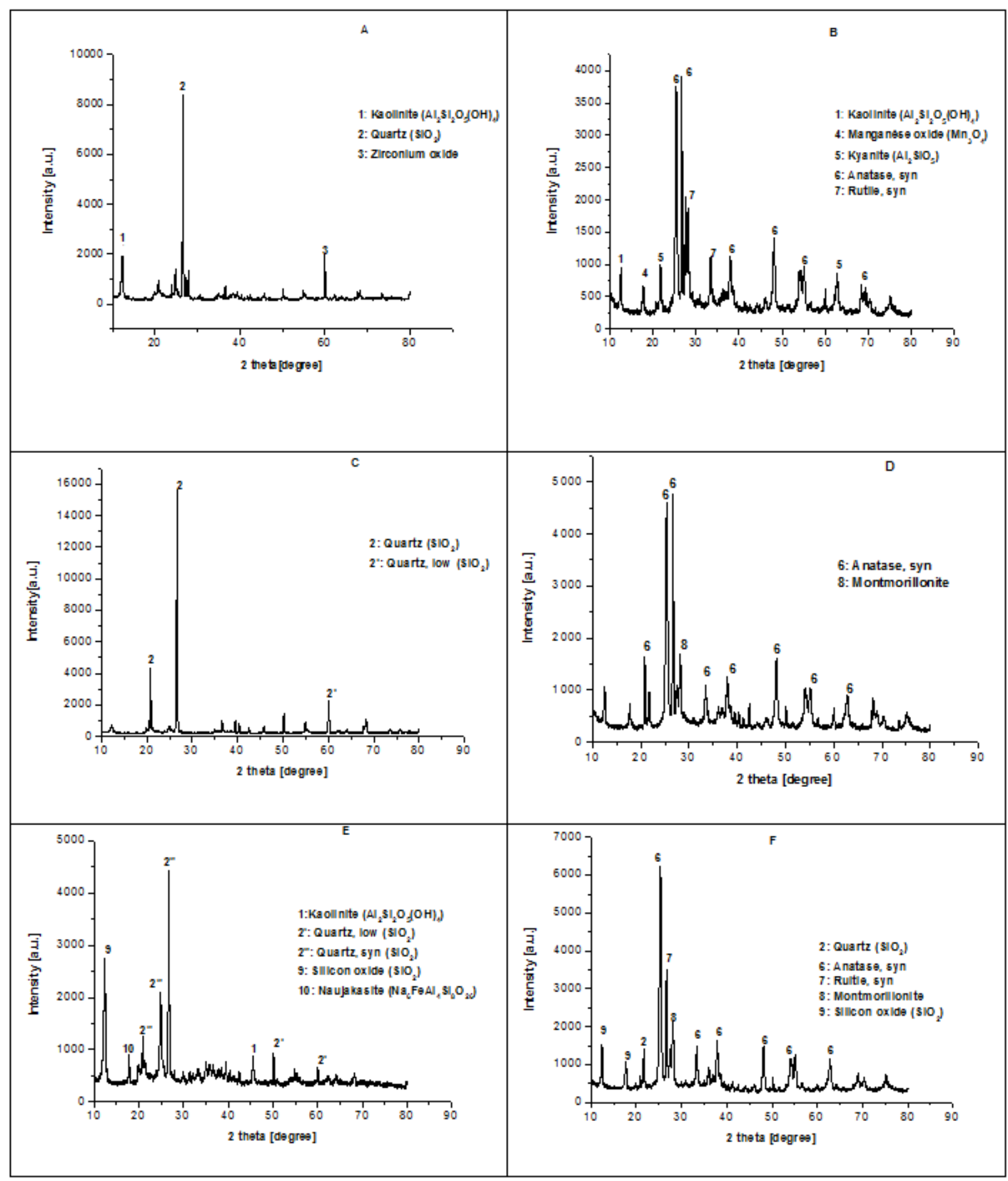

Figure 1: XRD graphs: (A) BW; (B) B-TiO $;$; (C) VW; (D)V- $\mathrm{TiO}_{2}$, (E) RW and (F ) R- $\mathrm{TiO}_{2}$.

of $\mathrm{B}-\mathrm{TiO}_{2}$ substrate (kyanite $\left(\mathrm{Al}_{2} \mathrm{SiO}_{5}\right)$ ), confirming that chemical restructuring occurs within $\mathrm{B}-\mathrm{W}$ when hydrothermally processed. The crystalline modifications are accompanied by a significant increase in the BET surface, from $19.35 \mathrm{~m}^{2} / \mathrm{g}$ in $\mathrm{B}-\mathrm{W}$ to $43.37 \mathrm{~m}^{2} / \mathrm{g}$ in $\mathrm{B}-\mathrm{TiO}_{2}$; from
$25.38 \mathrm{~m}^{2} / \mathrm{g}$ in $\mathrm{V}-\mathrm{W}$ to $44.29 \mathrm{~m}^{2} / \mathrm{g}$ in $\mathrm{V}-\mathrm{TiO} 2$ and $15.26 \mathrm{~m}^{2} / \mathrm{g} \mathrm{R}-\mathrm{W}$ to $32.88 \mathrm{~m}^{2} / \mathrm{g}$ in $\mathrm{R}-\mathrm{TiO}_{2}$.

Information on the new substrates (morphology/topography) was obtained from AFM and SEM micrographs (Figures 2-4). The AFM 
Citation: Guillaume PLA, Chelaru AM, Visa M, Lassiné O (2018) “Titanium Oxide-Clay” as Adsorbent and Photocatalysts for Wastewater Treatment. J Membra Sci Technol 8: 176. doi:10.4172/2155-9589.1000176

Page 4 of 11

\begin{tabular}{|c|c|c|c|c|}
\hline $2 \theta$ & COD/PDF & Crystalline phase & $\begin{array}{l}\text { Structures of } \\
\text { the crystallite }\end{array}$ & $\begin{array}{c}\text { Crystallite } \\
\text { size }(\AA)\end{array}$ \\
\hline \multicolumn{5}{|l|}{$B-W$} \\
\hline 12.262 & 00-001-0527 & Kaolinite $\left(\mathrm{Al}_{2} \mathrm{Si}_{2} \mathrm{O}_{5}(\mathrm{OH})_{4}\right)$ & triclinic & 135 \\
\hline 20.835 & 00-020-0452 & $\begin{array}{c}\text { Gismondine } \\
\left(\mathrm{CaAl}_{2} \mathrm{Si}_{2} \mathrm{O}_{8}: 4 \mathrm{H}_{2} \mathrm{O}\right)\end{array}$ & monoclinic & 285.9 \\
\hline 26.682 & 01-070-3755 & Quartz $\left(\mathrm{SiO}_{2}\right)$ & hexagonal & 455.9 \\
\hline 60.12 & 00-003-0640 & Zirconium oxide $\left(\mathrm{ZrO}_{2}\right)$ & cubic & 849 \\
\hline \multicolumn{5}{|l|}{$\mathrm{B}_{\mathrm{TiO}}$} \\
\hline 12.4 & 01-075-1593 & Kaolinite $\left(\mathrm{Al}_{2} \mathrm{Si}_{2} \mathrm{O}_{5}(\mathrm{OH})_{4}\right)$ & triclinic & 490.3 \\
\hline 17.543 & 00-004-0732 & Manganèse oxide $\left(\mathrm{Mn}_{3} \mathrm{O}_{4}\right)$ & cubic & 302.9 \\
\hline 21.72 & 01-073-3462 & Silicon oxide $\left(\mathrm{SiO}_{2}\right)$ & cubic & 294.3 \\
\hline 25.371 & $01-086-1157$ & Anatse, syn $\left(\mathrm{Ti}_{0}{ }^{\prime}{ }_{22} \mathrm{O}_{2}\right)$ & tetragonal & 237.7 \\
\hline 28.057 & 00-003-0513 & Kyanite $\left(\mathrm{Al}_{2} \mathrm{SiO}_{5}\right)$ & triclinic & 87.7 \\
\hline 33.438 & 01-073-3462 & Silicon oxide $\left(\mathrm{SiO}_{2}\right)$ & cubic & 294.3 \\
\hline 36.171 & 01-089-0553 & Rutile, syn $\left(\mathrm{Ti}_{0.912} \mathrm{O}_{2}\right)$ & tetragonal & 156.8 \\
\hline 38 & 01-086-1157 & Anatse, syn $\left(\mathrm{Ti}_{0,72} \mathrm{O}_{2}\right)$ & tetragonal & 237.7 \\
\hline 41.257 & 01-089-0553 & Rutile, syn $\left(\mathrm{Ti}_{0,912} \mathrm{O}_{2}\right)$ & tetragonal & 156.8 \\
\hline 48.114 & 01-086-1157 & Anatse, syn $\left(\mathrm{Ti}_{0,72} \mathrm{O}_{2}\right)$ & tetragonal & 237.7 \\
\hline 54 & 01-086-1157 & Anatse, syn $\left(\mathrm{Ti}_{0,72} \mathrm{O}_{2}\right)$ & tetragonal & 237.7 \\
\hline 55.142 & 01-086-1175 & Anatse, syn $\left(\mathrm{Ti}_{0,72} \mathrm{O}_{2}\right)$ & tetragonal & 237.7 \\
\hline 56.628 & 01-089-0553 & Rutile, syn $\left(\mathrm{Ti}_{0,912} \mathrm{O}_{2}\right)$ & tetragonal & 156.8 \\
\hline 60.114 & 00-016-0895 & Iron oxide $\left(\mathrm{Fe}_{2} \mathrm{O}_{3}\right)$ & & 181.4 \\
\hline 62.742 & 01-086-1157 & Anatse, syn $\left(\mathrm{Ti}_{0,72} \mathrm{O}_{2}\right)$ & tetragonal & 237.7 \\
\hline 68.457 & $00-003-0513$ & Kyanite $\left(\mathrm{Al}_{2} \mathrm{SiO}_{5}\right)$ & triclinic & 87.7 \\
\hline 69.028 & 01-075-1751 & Rutile $\left(\mathrm{TiO}_{2}\right)$ & tetragonal & 120.7 \\
\hline 75.199 & 01-086-1157 & Anatse, syn $\left(\mathrm{Ti}_{0,72} \mathrm{O}_{2}\right)$ & tetragonal & 237.7 \\
\hline
\end{tabular}

Table 1: The composition of the crystalline phases, $\mathrm{B}-\mathrm{W}$ and $\mathrm{B}-\mathrm{TiO}_{2}$.

images (only for $\mathrm{BW}$ and $\mathrm{B}-\mathrm{TiO}_{2}$.) and pore size distributions show different granular shapes. The highest roughness value $(120.712 \mathrm{~nm})$ corresponds to the new composites which has more aggregates with different, almost round and stable shapes (Figure 4). The composite has a large specific surface (larger than Degussa P25, $50.3 \mathrm{~nm}$ ), with small micro-pores (large enough to accommodate the dyes). The re-organizing process is confirmed by the AFM pictures, outlining significant differences between the randomly structured surfaces of clay washed (Figure 2) and the regular aggregates with droplet shape (Figure 3) assembled in rough structure that leave open macro-pores on the clay: $\mathrm{TiO}_{2}$ surface.

The predominant polar/ionic surface energy corresponds, in an oxide material in alkaline $\mathrm{pH}$ (larger than the point of zero charge), to a negatively charged surface and shows that the mild hydrothermal process increased the surface polarity/ionic degree. This combination of increased specific surface, homogeneity and roughness, and negative charges supports the use of the clay $\mathrm{TiO}_{2}$ composite as substrate in adsorption processes of neutral or cationic species [16]. Additional surface investigations were done and the SEM images are presented in Figure 4 . The SEM images confirm that the clay grains are cracked and micro-restructuring occur with significant modification of the surface aspect, as results of dissolution/re-precipitation reactions and $\mathrm{TiO}_{2}$ particles are embedded on surface of the clay.

The FT-IR spectra analyses of the composites $\mathrm{B}-\mathrm{TiO}_{2}, \mathrm{~V}-\mathrm{TiO} 2$ and $\mathrm{R}-\mathrm{TiO}_{2}$ synthesized are displayed in Figure 5 . The spectra analysis conducted to investigate the vibration frequency changes of the functional groups in the adsorbents materials (functional groups), indicating the complex nature of the adsorbent as shown in Figure 5 and in Table 4.

\section{Adsorption and photocatalytic processes on composites}

The active species generated by photo-irradiation can attack the pollutant, if this is in the very close vicinity of the substrate. Thus,

\begin{tabular}{|c|c|c|c|c|}
\hline $2 \theta$ & COD/PDF & Crystalline phase & $\begin{array}{l}\text { Structures of } \\
\text { the crystallite }\end{array}$ & $\begin{array}{c}\text { Crystallite } \\
\text { size }(\AA)\end{array}$ \\
\hline \multicolumn{5}{|l|}{$V-W$} \\
\hline 12.318 & $01-073-3410$ & Silicon oxide $\left(\mathrm{SiO}_{2}\right)$ & cubic & 119.7 \\
\hline 19.925 & $00-038-0360$ & Moganite $\left(\mathrm{SiO}_{2}\right)$ & Monoclinic & 243.9 \\
\hline 20.436 & $00-052-1379$ & Silicon oxide $\left(\mathrm{SiO}_{2}\right)$ & & 81.3 \\
\hline 20.833 & 01-070-7344 & Quartz $\left(\mathrm{SiO}_{2}\right)$ & Hexagonal & 480.9 \\
\hline 24.92 & 00-056-0505 & Silicon oxide $\left(\mathrm{SiO}_{2}\right)$ & Orthorhombic & 81.6 \\
\hline 26.623 & 01-070-7344 & Quartz $\left(\mathrm{SiO}_{2}\right)$ & Hexagonal & 480.9 \\
\hline 36.614 & 01-070-7344 & Quartz $\left(\mathrm{SiO}_{2}\right)$ & Hexagonal & 480.9 \\
\hline 39.509 & 01-070-7344 & Quartz $\left(\mathrm{SiO}_{2}\right)$ & Hexagonal & 480.9 \\
\hline 40.304 & 01-070-7344 & Quartz $\left(\mathrm{SiO}_{2}\right)$ & Hexagonal & 480.9 \\
\hline 42.518 & 01-070-7344 & Quartz $\left(\mathrm{SiO}_{2}\right)$ & Hexagonal & 480.9 \\
\hline 45.811 & 01-070-7344 & Quartz $\left(\mathrm{SiO}_{2}\right)$ & Hexagonal & 480.9 \\
\hline 50.182 & 01-070-7344 & Quartz $\left(\mathrm{SiO}_{2}\right)$ & Hexagonal & 480.9 \\
\hline 54.893 & 01-070-7344 & Quartz $\left(\mathrm{SiO}_{2}\right)$ & Hexagonal & 480.9 \\
\hline 60.059 & $00-005-0490$ & Quartz,low $\left(\mathrm{SiO}_{2}\right)$ & Hexagonal & 438.8 \\
\hline 64.033 & $00-005-0490$ & Quartz,low $\left(\mathrm{SiO}_{2}\right)$ & Hexagonal & 438.8 \\
\hline 67.779 & $00-005-0490$ & Quartz,low $\left(\mathrm{SiO}_{2}\right)$ & Hexagonal & 438.8 \\
\hline 68.46 & 01-070-7344 & Quartz $\left(\mathrm{SiO}_{2}\right)$ & Hexagonal & 480.9 \\
\hline 73.513 & $00-005-0490$ & Quartz,low $\left(\mathrm{SiO}_{2}\right)$ & Hexagonal & 438.8 \\
\hline 75.716 & 00-005-0490 & Quartz,low $\left(\mathrm{SiO}_{2}\right)$ & Hexagonal & 438.8 \\
\hline \multicolumn{5}{|l|}{$\mathrm{V}-\mathrm{TiO}_{2}$} \\
\hline 12.404 & 01-075-1593 & $\begin{array}{c}\text { Kaolinite } \\
\left(\mathrm{Al}_{2} \mathrm{Si}_{2} \mathrm{O}_{5}(\mathrm{OH})_{4}\right)\end{array}$ & triclinic & 439.4 \\
\hline 12.519 & 01-073-3442 & Silicon oxide $\left(\mathrm{SiO}_{2}\right)$ & tetragonal & 218.8 \\
\hline 17.72 & 01-073-3442 & Silicon oxide $\left(\mathrm{SiO}_{2}\right)$ & tetragonal & 218.8 \\
\hline 20.864 & $01-070-3755$ & Quartz $\left(\mathrm{SiO}_{2}\right)$ & Hexagonal & 584 \\
\hline 21.722 & 01-073-3462 & Silicon oxide (SiO2) & cubic & 264.7 \\
\hline 25.323 & 01-086-1157 & Anatse, syn $\left(\mathrm{Ti}_{0.72} \mathrm{O}_{2}\right)$ & tetragonal & 235.8 \\
\hline 26.638 & 01-070-3755 & Quartz (SiO2) & Hexagonal & 584 \\
\hline 27.436 & 01-089-0553 & Rutile, syn $\left(\mathrm{Ti}_{0,912} \mathrm{O}_{2}\right)$ & tetragonal & 227.2 \\
\hline 27.438 & $01-073-3460$ & Silicon oxide $\left(\mathrm{SiO}_{2}\right)$ & Monoclinic & 81.4 \\
\hline 28.122 & 00-011-0303 & Montmorillonite & & 177.1 \\
\hline 33.383 & 01-073-3442 & Silicon oxide $\left(\mathrm{SiO}_{2}\right)$ & cubic & 264.7 \\
\hline 36.07 & $00-001-1292$ & Rutile $\left(\mathrm{TiO}_{2}\right)$ & tetragonal & 280.3 \\
\hline 36.984 & 01-086-1157 & Anatse, syn $\left(\mathrm{Ti}_{0,72} \mathrm{O}_{2}\right)$ & tetragonal & 235.8 \\
\hline 37.899 & $01-086-1157$ & Anatse, syn $\left(\mathrm{Ti}_{0,72} \mathrm{O}_{2}\right)$ & tetragonal & 235.8 \\
\hline 37.954 & $00-042-1316$ & Ramdellite $\left(\mathrm{MnO}_{2}\right)$ & orthorhombic & 437.8 \\
\hline 38.585 & $01-086-1157$ & Anatse, syn $\left(\mathrm{Ti}_{0,72} \mathrm{O}_{2}\right)$ & tetragonal & 235.8 \\
\hline 39.442 & 01-070-3755 & Quartz $\left(\mathrm{SiO}_{2}\right)$ & Hexagonal & 584 \\
\hline 40.297 & 01-073-1117 & Titanium oxide $\left(\mathrm{Ti}_{3} \mathrm{O}_{5}\right)$ & Hexagonal & 190.2 \\
\hline 40.3 & 01-070-3555 & Quartz $\left(\mathrm{SiO}_{2}\right)$ & Hexagonal & 584 \\
\hline 41.214 & $00-001-1292$ & Rutile $\left(\mathrm{TiO}_{2}\right)$ & tetragonal & 280.3 \\
\hline 42.415 & 01-070-3755 & Quartz $\left(\mathrm{SiO}_{2}\right)$ & Hexagonal & 584 \\
\hline 44.129 & $00-001-1292$ & Rutile $\left(\mathrm{TiO}_{2}\right)$ & tetragonal & 280.3 \\
\hline 48.047 & 01-086-1157 & Anatse, syn $\left(\mathrm{Ti}_{0.72} \mathrm{O}_{2}\right)$ & tetragonal & 235.8 \\
\hline 50.132 & 01-070-3755 & Quartz $\left(\mathrm{SiO}_{2}\right)$ & Hexagonal & 584 \\
\hline 50.184 & 00-013-0458 & $\begin{array}{l}\text { Maghemite, } \mathrm{Q} \text { syn } \\
\left(\mathrm{Fe}_{2} \mathrm{O}_{3}\right)\end{array}$ & tetragonal & 914.4 \\
\hline 53.961 & 01-086-1157 & Anatse, syn $\left(\mathrm{Ti}_{0,72} \mathrm{O}_{2}\right)$ & tetragonal & 235.8 \\
\hline 54.015 & $00-042-1316$ & Ramdellite $\left(\mathrm{MnO}_{2}\right)$ & orthorhombic & 437.8 \\
\hline 55.105 & 01-086-1157 & Anatse, syn $\left(\mathrm{Ti}_{0,72} \mathrm{O}_{2}\right)$ & tetragonal & 235.8 \\
\hline 56.645 & 01-089-0553 & Rutile, syn $\left(\mathrm{Ti}_{0,912} \mathrm{O}_{2}\right)$ & tetragonal & 227.2 \\
\hline 59.964 & 01-070-3755 & Quartz $\left(\mathrm{SiO}_{2}\right)$ & Hexagonal & 584 \\
\hline 60.074 & 00-018-0803 & Manganèse $\left(\mathrm{Mn}_{3} \mathrm{O}_{4}\right)$ & tetragonal & 227.7 \\
\hline 62.761 & 00-004-0755 & $\begin{array}{l}\text { Maghemite, syn } \\
\qquad\left(\mathrm{Fe}_{2} \mathrm{O}_{3}\right)\end{array}$ & cubic & 183.8 \\
\hline 62.764 & $01-086-1157$ & Anatse, syn $\left(\mathrm{Ti}_{0,72} \mathrm{O}_{2}\right)$ & tetragonal & 235.8 \\
\hline 68.881 & $01-086-1157$ & Anatse, syn $\left(\mathrm{Ti}_{0.72} \mathrm{O}_{2}\right)$ & tetragonal & 235.8 \\
\hline 68.991 & $00-001-1292$ & Rutile $\left(\mathrm{TiO}_{2}\right)$ & tetragonal & 280.3 \\
\hline 70.31 & $01-086-1157$ & Anatse, syn $\left(\mathrm{Ti}_{0.72} \mathrm{O}_{2}\right)$ & tetragonal & 235.8 \\
\hline 75.169 & $01-086-1157$ & Anatse, syn $\left(\mathrm{Ti}_{0.72} \mathrm{O}_{2}\right)$ & tetragonal & 235.8 \\
\hline
\end{tabular}

Table 2: The composition of the crystalline phases, $\mathrm{V}-\mathrm{W}$ and $\mathrm{V}-\mathrm{TiO}_{2}$. 


\begin{tabular}{|c|c|c|c|c|}
\hline $2 \theta$ & COD/PDF & Crystalline phase & $\begin{array}{l}\text { Structures of } \\
\text { the crystallite }\end{array}$ & $\begin{array}{c}\text { Crystallite } \\
\text { size }(\AA)\end{array}$ \\
\hline \multicolumn{5}{|l|}{$R-W$} \\
\hline 12.317 & 01-073-3410 & Silicon oxide $\left(\mathrm{SiO}_{2}\right)$ & cubic & 139.6 \\
\hline 17.827 & 01-070-187 & $\begin{array}{c}\text { Naujakasite } \\
\left(\mathrm{Na}_{6} \mathrm{FeAl}_{4} \mathrm{Si}_{8} \mathrm{O}_{26}\right)\end{array}$ & Monoclinic & 81.5 \\
\hline 20.887 & 00-033-1161 & Quartz, syn $\left(\mathrm{SiO}_{2}\right)$ & Hexagonal & 355.8 \\
\hline 26.676 & $00-033-1161$ & Quartz, syn $\left(\mathrm{SiO}_{2}\right)$ & Hexagonal & 335.8 \\
\hline 30.03 & 01-085-1369 & $\begin{array}{c}\text { Grossular, Ferrian } \\
\left(\mathrm{Ca}_{3} \mathrm{Al}_{1,332} \mathrm{Fe}_{0,668} \mathrm{Si}_{3} \mathrm{O}_{12}\right)\end{array}$ & Orthorhombic & 1072.4 \\
\hline 33.26 & 00-013-0534 & Hematite, syn $\left(\mathrm{Fe}_{2} \mathrm{O}_{3}\right)$ & Rhombo.H.axe & 103.4 \\
\hline 33.326 & $00-052-1560$ & $\begin{array}{l}\text { Cronstedile-2H2 } \\
\left(\mathrm{Fe}_{3}\left(\mathrm{Si}_{2} \mathrm{O}_{5}\right)(\mathrm{OH})_{4}\right)\end{array}$ & Orthorhombic & 134.9 \\
\hline 34.963 & 00-011-0474 & Titanium oxide $\left(\mathrm{Ti}_{10} \mathrm{O}_{19}\right)$ & & 129.6 \\
\hline 35.071 & $00-18-1404$ & Titanium oxide $\left(\mathrm{Ti}_{8} \mathrm{O}_{15}\right)$ & Triclinic & 82.9 \\
\hline 35.077 & 00-056-1303 & Iron oxide $\left(\mathrm{Fe}_{2} \mathrm{O}_{3}\right)$ & Orthorhombic & 256.2 \\
\hline 35.824 & 01-085-0514 & Calcium peroxide $\left(\mathrm{CaO}_{2}\right)$ & tetragonal & 817.5 \\
\hline 35.871 & $00-18-1405$ & Titanium oxide $\left(\mathrm{Ti}_{9} \mathrm{O}_{15}\right)$ & Triclinic & 95.8 \\
\hline 37.858 & 01-082-1570 & Silicon oxide $\left(\mathrm{SiO}_{2}\right)$ & Orthorhombic & 81.7 \\
\hline 45.589 & 00-001-0527 & Kaolinite $\left(\mathrm{Al}_{2} \mathrm{Si}_{2} \mathrm{O}_{5}(\mathrm{OH})_{4}\right)$ & Triclinic & 139.7 \\
\hline 50.174 & 00-005-0490 & Quartz, low $\left(\mathrm{SiO}_{2}\right)$ & Hexagonal & 336.3 \\
\hline 50.188 & 00-019-0231 & $\begin{array}{c}\text { Tungusite } \\
\left(\mathrm{Ca}_{4} \mathrm{Fe}_{2} \mathrm{Si}_{6} \mathrm{O}_{15}(\mathrm{OH})_{6}\right)\end{array}$ & & 737 \\
\hline 50.3 & 00-018-1205 & $\begin{array}{l}\text { Sodium Calcium } \\
\text { Hydroxide Silicate }\end{array}$ & Monoclinic & 100.4 \\
\hline 59.994 & 00-005-0490 & Quartz, low $\left(\mathrm{SiO}_{2}\right)$ & Hexagonal & 336.3 \\
\hline 62.377 & 00-013-0162 & Manganèse $\left(\mathrm{Mn}_{3} \mathrm{O}_{4}\right)$ & cubic & 184.2 \\
\hline 64.137 & $00-013-0534$ & Hematite, syn (Fe2O3) & Rhombo.H.axe & 103.4 \\
\hline 68.298 & 00.042 .1468 & Corundum, syn & Rhombo.H.axe & 277.8 \\
\hline \multicolumn{5}{|l|}{$\mathrm{R}-\mathrm{TiO}_{2}$} \\
\hline 12.346 & $01-078-2110$ & Kaolinite $\left(\mathrm{Al}_{2} \mathrm{Si}_{2} \mathrm{O}_{5}(\mathrm{OH})_{4}\right)$ & triclinic & 144.5 \\
\hline 12.518 & 01-073-3442 & Silicon oxide $\left(\mathrm{SiO}_{2}\right)$ & Tetragonal & 204.3 \\
\hline 17.719 & 01-073-3442 & Silicon oxide $\left(\mathrm{SiO}_{2}\right)$ & Tetragonal & 204.3 \\
\hline 19.891 & 00-011-0303 & Montmorillonite & & 207.1 \\
\hline 20.863 & $01-070-7433$ & Quartz $\left(\mathrm{SiO}_{2}\right)$ & Hexagonal & 441.1 \\
\hline 21.72 & 01-073-3462 & Silicon oxide $\left(\mathrm{SiO}_{2}\right)$ & cubic & 298.4 \\
\hline 25.322 & 01-086-1157 & Anatse, syn $\left(\mathrm{Ti}_{0,72} \mathrm{O}_{2}\right)$ & Tetragonal & 272.9 \\
\hline 26.636 & 01-070-7344 & Quartz $\left(\mathrm{SiO}_{2}\right)$ & Hexagonal & 441.1 \\
\hline 27.436 & 03-065-0191 & Rutile, syn $\left(\mathrm{O}_{2} \mathrm{Ti}\right)$ & Tetragonal & 269.9 \\
\hline 28.065 & $00-003-0513$ & Kyanite $\left(\mathrm{Al}_{2} \mathrm{SiO}_{5}\right)$ & triclinic & 183.3 \\
\hline 28.11 & $00-001-0303$ & Montmorillonite & & 207.1 \\
\hline 36.962 & 01-086-1157 & Anatse, syn $\left(\mathrm{Ti}_{0,72} \mathrm{O}_{2}\right)$ & Tetragonal & 272.9 \\
\hline 37.897 & 01-086-1157 & Anatse, syn $\left(\mathrm{Ti}_{0,72} \mathrm{O}_{2}\right)$ & Tetragonal & 272.9 \\
\hline 39.497 & 00-033-1161 & Quartz, syn $\left(\mathrm{SiO}_{2}\right)$ & Hexagonal & 449.3 \\
\hline 46.127 & 01-073-3462 & Silicon oxide $\left(\mathrm{SiO}_{2}\right)$ & cubic & 298.4 \\
\hline 48.67 & 01-086-1157 & Anatse, syn $\left(\mathrm{Ti}_{0,72} \mathrm{O}_{2}\right)$ & Tetragonal & 272.9 \\
\hline 54.011 & 01-086-1157 & Anatse, syn $\left(\mathrm{Ti}_{0,72} \mathrm{O}_{2}\right)$ & Tetragonal & 272.9 \\
\hline 55.101 & 01-086-1157 & Anatse, syn $\left(\mathrm{Ti}_{0,72} \mathrm{O}_{2}\right)$ & Tetragonal & 272.9 \\
\hline 56.645 & 03-065-0191 & Rutile, syn $\left(\mathrm{O}_{2} \mathrm{Ti}\right)$ & Tetragonal & 269.9 \\
\hline 59.96 & 01.070 .7344 & Quartz $\left(\mathrm{SiO}_{2}\right)$ & Hexagonal & 441.1 \\
\hline 62.761 & 01-086-1157 & Anatse, syn $\left(\mathrm{Ti}_{0,72} \mathrm{O}_{2}\right)$ & Tetragonal & 272.9 \\
\hline 64.075 & 00-013-0534 & Hematite, syn $\left(\mathrm{Fe}_{2} \mathrm{O}_{3}\right)$ & Rhombo.H.axe & 363.7 \\
\hline 68.877 & 01-086-1157 & Anatse, syn $\left(\mathrm{Ti}_{0,72} \mathrm{O}_{2}\right)$ & Tetragonal & 272.9 \\
\hline 70.306 & 01-086-1157 & Anatse, syn $\left(\mathrm{Ti}_{0,72} \mathrm{O}_{2}\right)$ & Tetragonal & 272.9 \\
\hline 75.164 & 01-086-1157 & Anatse, syn $\left(\mathrm{Ti}_{0,72} \mathrm{O}_{2}\right)$ & Tetragonal & 272.9 \\
\hline
\end{tabular}

Table 3: The composition of the crystalline phases, $\mathrm{R}-\mathrm{W}$ and $\mathrm{R}-\mathrm{TiO}_{2}$.

adsorbed pollutant species will be removed efficiently by photocatalytic processes. Studies are already reported linking the pollutants' structures, adsorption and the photocatalytic efficiency $[17,18]$. The most active photocatalytic component of the composite is $\mathrm{TiO}_{2}$ because the $\mathrm{TiO}_{2}$ polymorphs have band gaps of $3.0 \mathrm{eV}$ (rutile), $3.2 \mathrm{eV}$ (anatase) thus are active under UV radiation with wavelengths lower than 413 $\mathrm{nm}$ for rutile, $387 \mathrm{~nm}$ for anatase. As the UV wavelength used in our experiments was $365 \mathrm{~nm}$, we may conclude that only anatase and rutile are actually activated under irradiation and can exhibit the well-known coupling effect [19].

The solutions containing only $\mathrm{MB}(0.03125 \mathrm{mM})$ are well treated using all materials (Figure 5). But, the solutions containing $\mathrm{MB}$ and $\mathrm{Cd}^{2+}$, efficiencies of $\mathrm{MB}$ decrease because of an affinity orders of the species, towards the substrate: $\mathrm{Cd}^{2+}>\mathrm{MB}[20] . \mathrm{Cd}^{2+}$ is firstly adsorbed on the substrates and then MB. The chemical structure of MB is shown in Figure 6. The photocatalysis process efficiency after $360 \mathrm{~min}$ on materials are presented in Table 5.

The adsorption efficiency after $30 \mathrm{~min}$, for $\mathrm{MB}$ and $\mathrm{Cd}^{2+}$ removal on all composites are presented on Table 6 . Better results were obtained using $\mathrm{R}-\mathrm{TiO}_{2}$ material. The XRD graph of $\mathrm{R}-\mathrm{TiO}_{2}$ shows the presence of new compounds (montmorillonite) that have good adsorption proprieties [14].

\section{The adsorption mechanism}

In system containing two or more pollutants and the substrate, several adsorption processes develop/occur [13-16]:

a) $\mathrm{The} \mathrm{Cd}^{2+}$ can be absorbed by $\mathrm{Ti}-\mathrm{OH}$ of the layer, but with lower efficiency (Equation 3):

$$
\mathrm{Ti}-\mathrm{OH}+\mathrm{Cd}^{2+}\left(\mathrm{H}_{2} \mathrm{O}\right)_{\mathrm{n}} \Leftrightarrow \mathrm{Ti}-\mathrm{OCd}^{+}\left(\mathrm{H}_{2} \mathrm{O}\right)_{\mathrm{n}-\mathrm{x}}+\mathrm{H}^{+}\left(\mathrm{H}_{2} \mathrm{O}\right) \mathrm{x}
$$

b) In the multicomponent solutions, $\mathrm{Cd}^{2+}+\mathrm{MB}$ interaction can be developed, further influencing the adsorption rate and its mechanisms. Many organic substances like aromatic compounds are attached to the modified clay $\left(\mathrm{B}-\mathrm{TiO}_{2}, \mathrm{~V}-\mathrm{TiO}_{2}\right.$ and $\mathrm{R}-\mathrm{TiO}_{2}$ ) surface by hydrogen bonding, but stronger interactions with formation of new bonds can be observed for other molecules.

The possible reactions are proposed in Figure 7 involving the lone pair of electrons from the pyridine nitrogen atom or chlorine in $\mathrm{MB}$ molecules. Stereochemistry complexes with cadmium are determined only by the ionic volume strength of the strong electrostatic and covalent bonds. The volume effect makes the $\mathrm{Cd}^{2+}$ to be more apt to forms tetraor hexa-coordinated complexes with distorted octahedral structure Figure 8. These interactions/bonds can be correlated with FTIR spectra Figure 9. The peak at $605.64 \mathrm{~cm}-1$ of MB disappears, there. The Si-O vibration band is at $605.64 \mathrm{~cm}-1$ and disappears with $\mathrm{Cd}^{2+}$ in solution.

\section{The photo-fenton mechanism}

Under UV radiation the electron-hole pair is formed. The most common mechanisms involve the holes for hydroxyl radical production. In alkaline media several other reactions involving the $\mathrm{O}_{2} / \mathrm{HO}^{-}, \mathrm{O}_{2} /$ $\mathrm{HOO}^{-}$and the $\mathrm{O}_{2} / \mathrm{H}_{2} \mathrm{O}_{2}$ couples are possible $[21,22]$. Another undesired process is the electron-hole recombination. The photocatalytic efficiency strongly depends on the system's ability to limit this process. The hydrogen peroxide addition is expected to support electron trapping. In this work, in presence of $0.1 \mathrm{~g}$ composite $+10 \mu \mathrm{L} \mathrm{H}_{2} \mathrm{O}_{2}+50$ $\mathrm{mL}$ pollutant solution $+20 \mu \mathrm{L} \mathrm{Fe}{ }^{2+}$ (Fenton's reagent $\left(\mathrm{Fe}^{2+} / \mathrm{H}_{2} \mathrm{O}_{2}\right.$ ), there is an are increase in the efficiency of organic pollutants degradation (Figure 10). As exhibited in this Figure, a mixture of $\mathrm{H}_{2} \mathrm{O}_{2}$ and $\mathrm{Fe}^{2+}$ in medium with the range of $\mathrm{pH} 4-5$ has good oxidizing properties of the organic pollutants developing the hydroxyl radicals [23]. The classical mechanism is a simple redox reaction in which $\mathrm{Fe}^{2+}$ ions are oxidized to $\mathrm{Fe}^{3+}$ ions and the $\mathrm{H}_{2} \mathrm{O}_{2}$ is reduced to hydroxide ion and hydroxide radical (Equation 4). 

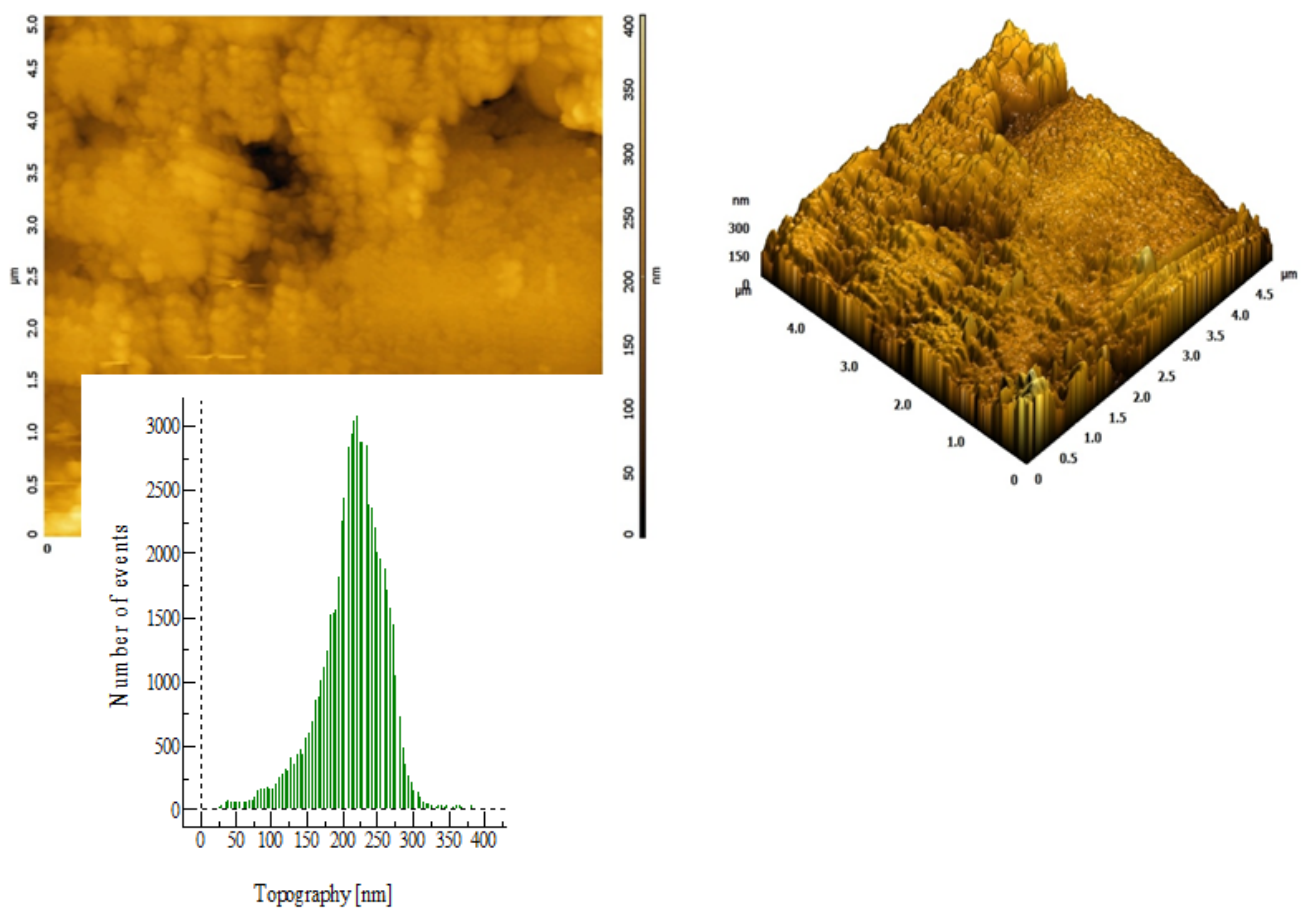

Figure 2: AFM topography of BW; Average roughness: $46.905 \mathrm{~nm}$.
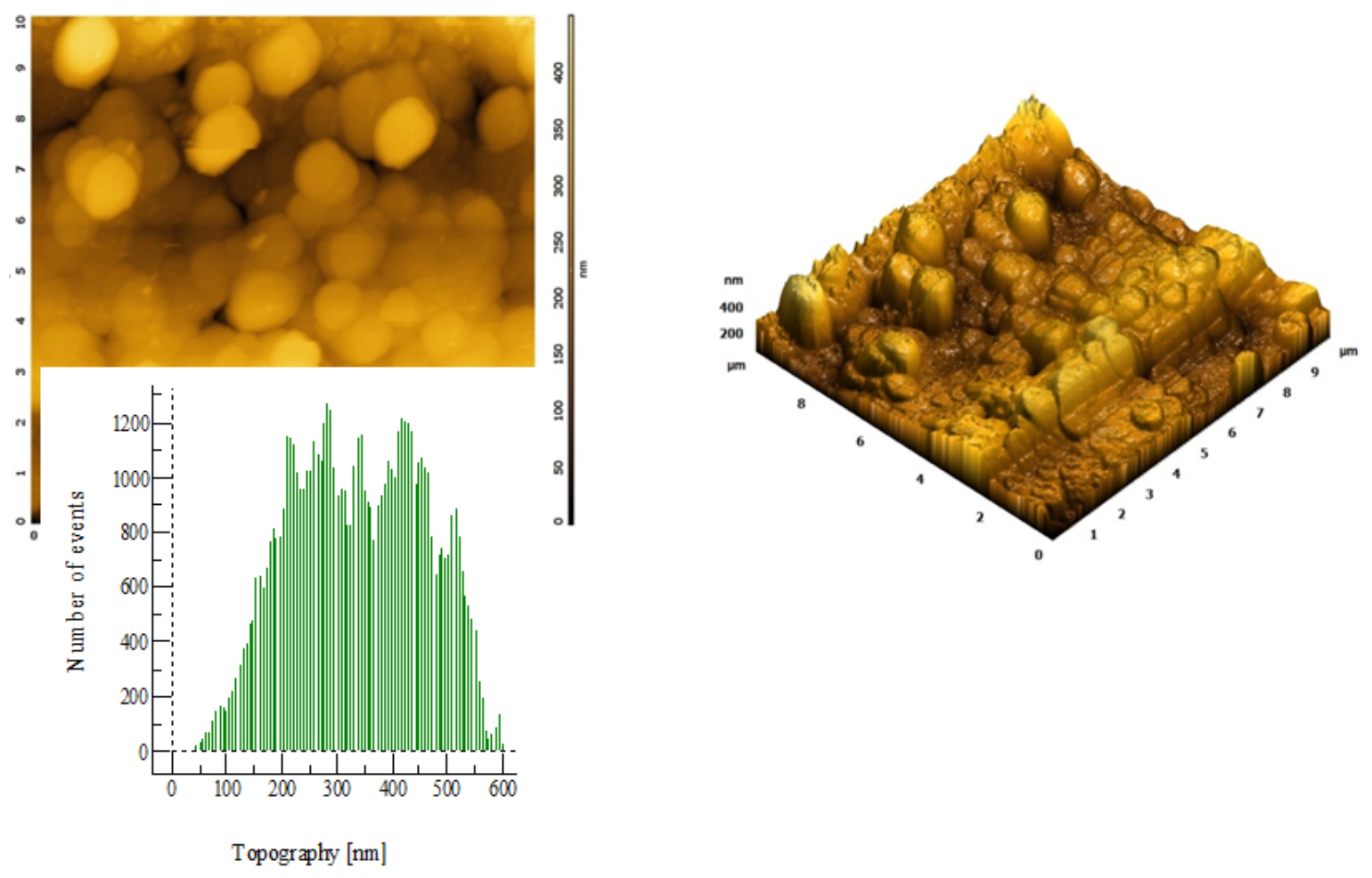

Figure 3: AFM topography of $\mathrm{B}-\mathrm{TiO}_{2}$; Average roughness: $120.712 \mathrm{~nm}$. 


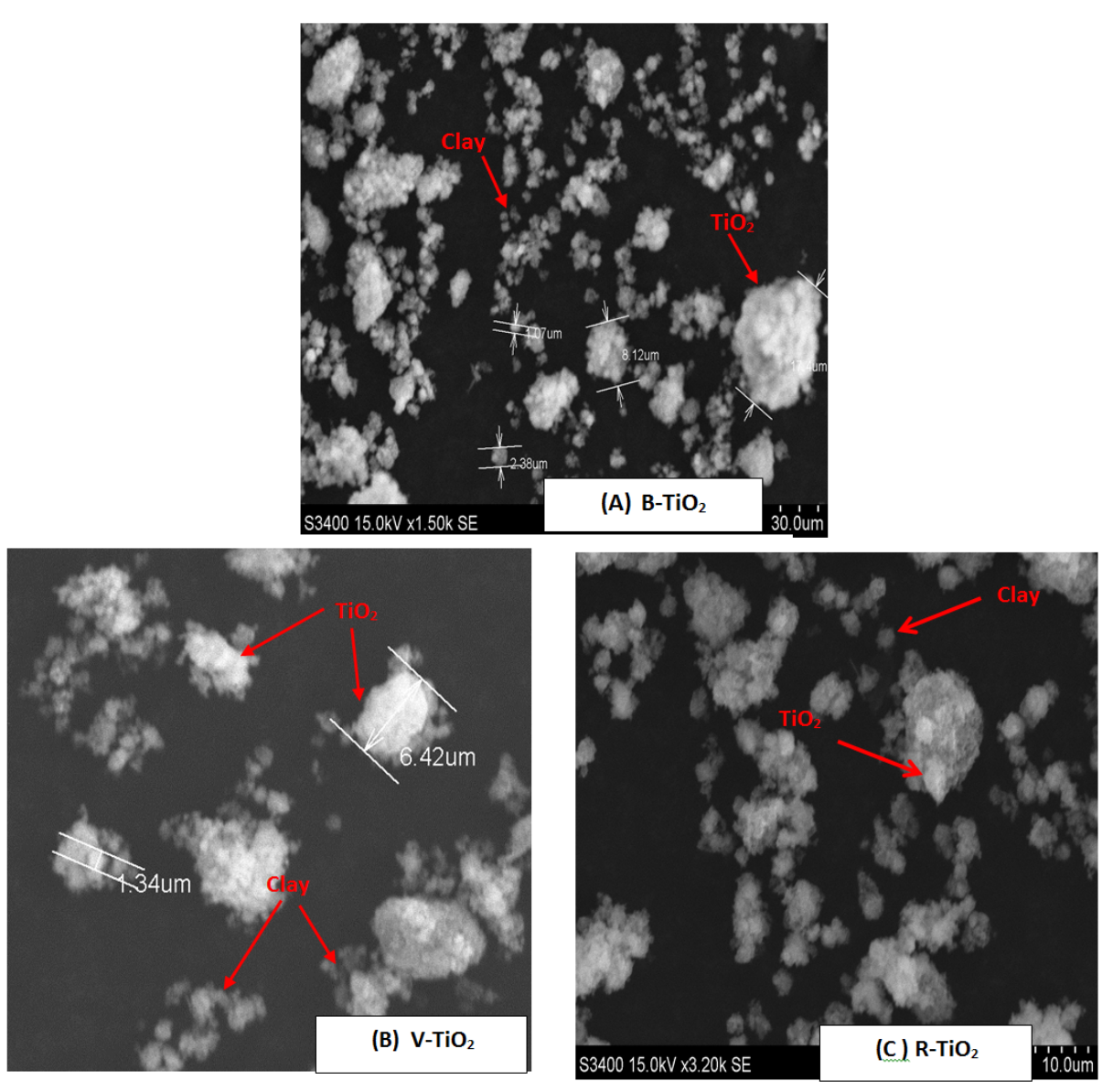

Figure 4: Scanning electron microscopic images of $(\mathrm{A}) \mathrm{B}-\mathrm{TiO}_{2} ;(\mathrm{B}) \mathrm{V}-\mathrm{TiO}_{2}$ and $(\mathrm{C}) \mathrm{R}-\mathrm{TiO}_{2}$

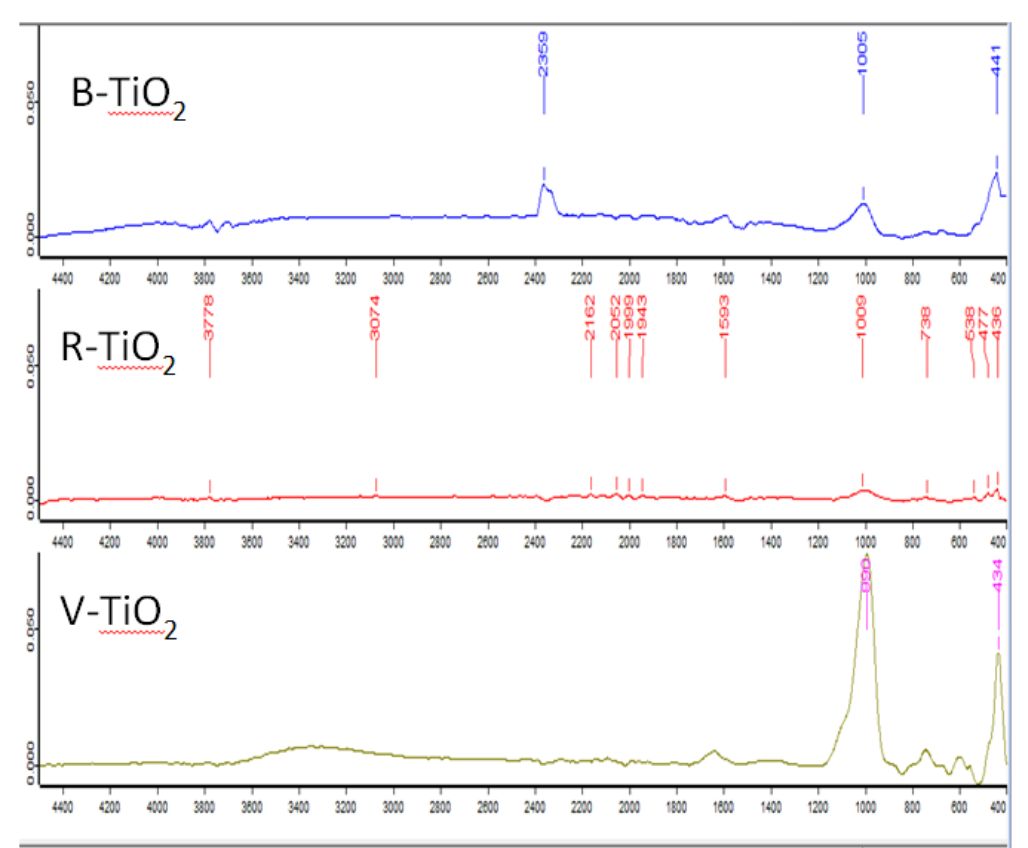

Figure 5: FT-IR spectra analyses of $\mathrm{B}-\mathrm{TiO}_{2}, \mathrm{~V}-\mathrm{TiO}_{2}$ and $\mathrm{R}-\mathrm{TiO}_{2}$ 


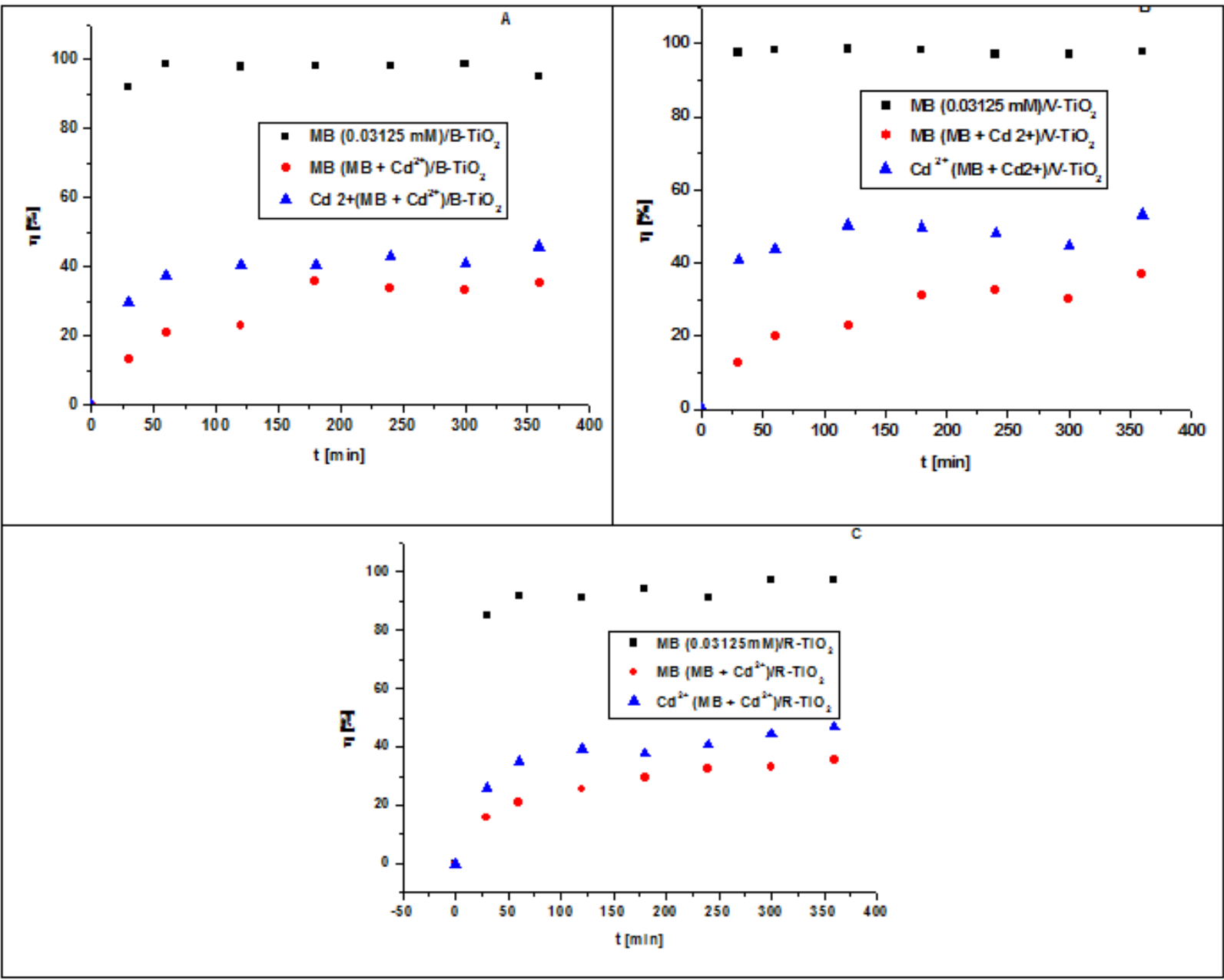

Figure 6: $\mathrm{MB}$ and $\mathrm{Cd}^{2+}$ photodegradation efficiency vs. time on $\mathrm{B}-\mathrm{TiO}_{2}(\mathrm{~A}), \mathrm{V}-\mathrm{TiO}_{2}(\mathrm{~B})$ and $\mathrm{R}-\mathrm{TiO}_{2}(\mathrm{C})$.

\begin{tabular}{|l|c|c|c|}
\hline Characteristics groups & $\begin{array}{c}\mathbf{B}-\mathrm{TiO}_{\mathbf{2}} \\
{\left[\mathbf{c m}^{-1}\right]}\end{array}$ & $\begin{array}{c}\mathbf{R}-\mathrm{TiO}_{2} \\
{\left[\mathbf{c m}^{-1}\right]}\end{array}$ & $\begin{array}{c}\mathbf{V}-\mathrm{TiO}_{2} \\
{\left[\mathbf{c m}^{-1}\right]}\end{array}$ \\
\hline $\begin{array}{l}\mathrm{Si}-\mathbf{( O H}) \mathbf{A l} \text { hydroxyl groups } \\
\text { stretching }\end{array}$ & 3719 & 3778 & 3789 \\
\hline $\begin{array}{l}\text { OH groups bridging hydroxyls } \\
\text { in zeolite cages to the same Al - } \\
\text { OH - Si }\end{array}$ & 3648 & - & $\begin{array}{c}3334.9 \text { with } \\
\text { shoulders } \\
3350\end{array}$ \\
\hline Adsorbed CO & & & 2320 \\
\hline Water molecules & 2359 & 2372 & 1637 \\
\hline Asymmetric stretch & 1603 & 1603 & 990 \\
\hline Ti - O - Si & 1005 & 1009 & 434 \\
\hline O-Ti-O from rutile & 441 & 434 & 727.8 \\
\hline Ti - O - Ti bridging vibration & 745.8 & 763 & 588 \\
\hline $\begin{array}{l}\text { Si - O bond of the zeolite } \\
\text { structure }\end{array}$ & 675 & 662 & \\
\hline
\end{tabular}

Table 4: The vibration frequency of the functional groups in the adsorbents materials.

\begin{tabular}{|l|c|c|c|}
\hline Materials/Samples & $\mathbf{B}-\mathrm{TiO}_{2}$ & $\mathbf{V}-\mathrm{TiO}_{2}$ & $\mathbf{R}-\mathrm{TiO}_{2}$ \\
\hline MB (0.03125 mM) & 98.75 & 97 & 97.48 \\
\hline $\mathbf{C d}^{2+}\left(\mathbf{M B}+\mathbf{C d}^{2+}\right)$ & 41.11 & 46.7 & 44.37 \\
\hline MB (MB + Cd $\left.{ }^{2+}\right)$ & 33.32 & 35.23 & 35.98 \\
\hline
\end{tabular}

Table 5: Photocatalysis process efficiency [\%] on substrates with $\mathrm{TiO}_{2}$ embedded.

\begin{tabular}{|c|c|c|c|}
\hline Materials / Samples & B-TiO2 & V-TiO2 & R-TiO2 \\
\hline MB (0.03125 $\mathbf{~ m M )}$ & 90.31 & 90.2 & 91.19 \\
\hline $\mathbf{C d}^{2+}$ (MB + $\mathbf{C d}^{\mathbf{2 +}}$ ) & 18.04 & 21.63 & 27.05 \\
\hline MB (MB + $\mathbf{C d}^{2+}$ ) & 26.6 & 30.38 & 30.45 \\
\hline
\end{tabular}

Table 6: Adsorption process efficiency [\%] on substrates.

$\mathrm{Fe}^{2+}{ }_{\mathrm{a}} \mathrm{q}+\mathrm{H}_{2} \mathrm{O}_{2} \rightarrow \mathrm{Fe}^{3+}{ }_{\mathrm{a}} \mathrm{q}+\mathrm{HO} \bullet+\mathrm{OH}$

The $\mathrm{Fe}^{3+}$ ion produced in reaction (4) can be reduced back to $\mathrm{Fe}^{2+}$ ion by a second molecule of hydrogen peroxide (equation 5 ).

$$
\mathrm{Fe}^{3+}{ }_{\mathrm{a}} \mathrm{q}+\mathrm{H}_{2} \mathrm{O}_{2}+\mathrm{H}_{2} \mathrm{O} \rightarrow \mathrm{Fe}^{2+}{ }_{\mathrm{a}} \mathrm{q}+\mathrm{H}_{3} \mathrm{O}^{+}+\mathrm{HO}_{2} \cdot
$$

The stoichiometry of Fenton degradation reactions is complex. In addition to $\mathrm{Fe}^{2+} / \mathrm{Fe}^{+}$and hydrogen peroxide, can involve the participation of the hydroperoxyl radical, HOO• iron (IV) or ferryl, $\mathrm{FeO}^{2+}$, dissolved molecular oxygen, organic hydroperoxides, and other intermediates formed during the degradation. Under irradiation of Fenton systems with UV light strongly accelerated the rate of degradation of a variety of organic pollutants for example $\mathrm{MB}$ dye. This behavior upon irradiation is due principally to the photochemical reduction of $\mathrm{Fe}^{3+} \rightarrow \mathrm{Fe}^{2+}$, for which the net reaction can be written as (Equation 6): [24] 


$$
\mathrm{Fe}^{3+}{ }_{a} q+\mathrm{H}_{2} \mathrm{O} \stackrel{h v}{\rightarrow} \mathrm{Fe}^{2+}{ }_{a} q+\mathrm{HO} \cdot+H^{+}
$$

The $\mathrm{Cd}^{2+}$ can be adsorbed and is activated by $\mathrm{H}_{2} \mathrm{O}_{2}$-corroborated with a partial dehydration (thus are higher mobility); the significant increase in the removal efficiency of $\mathrm{MB}$ in this condition (Equation 7):

$\mathrm{Ti}-\mathrm{OH}+\mathrm{Cd}^{2+}\left(\mathrm{H}_{2} \mathrm{O}\right)_{\mathrm{n}}+\mathrm{OH}^{\star} \Leftrightarrow \mathrm{Ti}-\mathrm{OCd}^{+}\left(\mathrm{H}_{2} \mathrm{O}\right)_{\mathrm{n}-\mathrm{x}}+\left(\mathrm{H}_{2} \mathrm{O}\right) \mathrm{x}+\mathrm{H}_{2} \mathrm{O}$

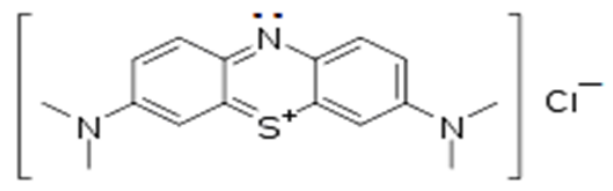

Figure 7: The chemical structure of Methylene Blue.
The photo-Fenton process efficiency, after $360 \mathrm{~min}$, on all materials is presented in Table 7 .

The kinetic studies: kinetic parameters of pollutants for adsorption and photodegradation

The kinetic parameters are best fitted by the pseudo-second order kinetic model [25], with the linear form given by Equation (8):

$$
\frac{t}{q_{t}}=\frac{1}{\left(k_{2} q_{e}^{2}\right)}+\frac{t}{q_{e}}
$$

Where:

$\mathrm{k}_{2}$ is the pseudo second-order rate constant $\left(\mathrm{g} \mathrm{mg}^{-1} \mathrm{~min}^{-1}\right)$ and can be evaluated from the slope of the plot. Based on Equation (8) the

\begin{tabular}{|c|c|c|c|}
\hline Materials / Samples & B-TiO2 & V-TiO2 & R-TiO2 \\
\hline MB (0.03125 $\mathbf{~ m M )}$ & 99.07 & 96.86 & 98.8 \\
\hline $\mathbf{C d}^{2+}\left(\mathbf{M B}+\mathbf{C d}^{2+}\right)$ & 56.39 & 58.21 & 62.56 \\
\hline MB (MB + $\mathbf{C d}^{2+}$ ) & 50.6 & 50.87 & 56.47 \\
\hline
\end{tabular}

Table 7: Photo-Fenton process efficiency [\%] on materials.

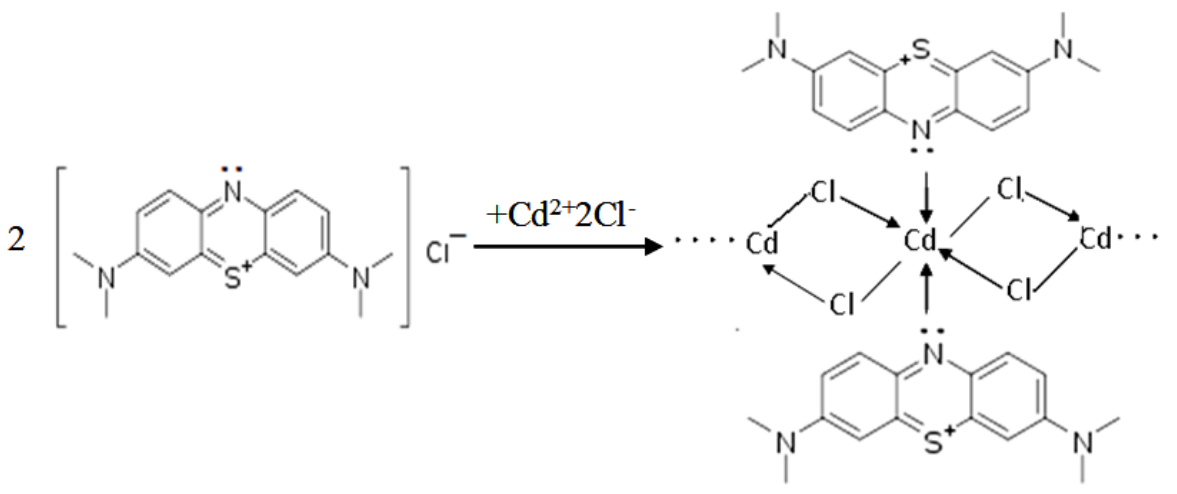

Figure 8: The interaction of $\mathrm{Cd}^{2+}$ with Methylene Blue molecule.

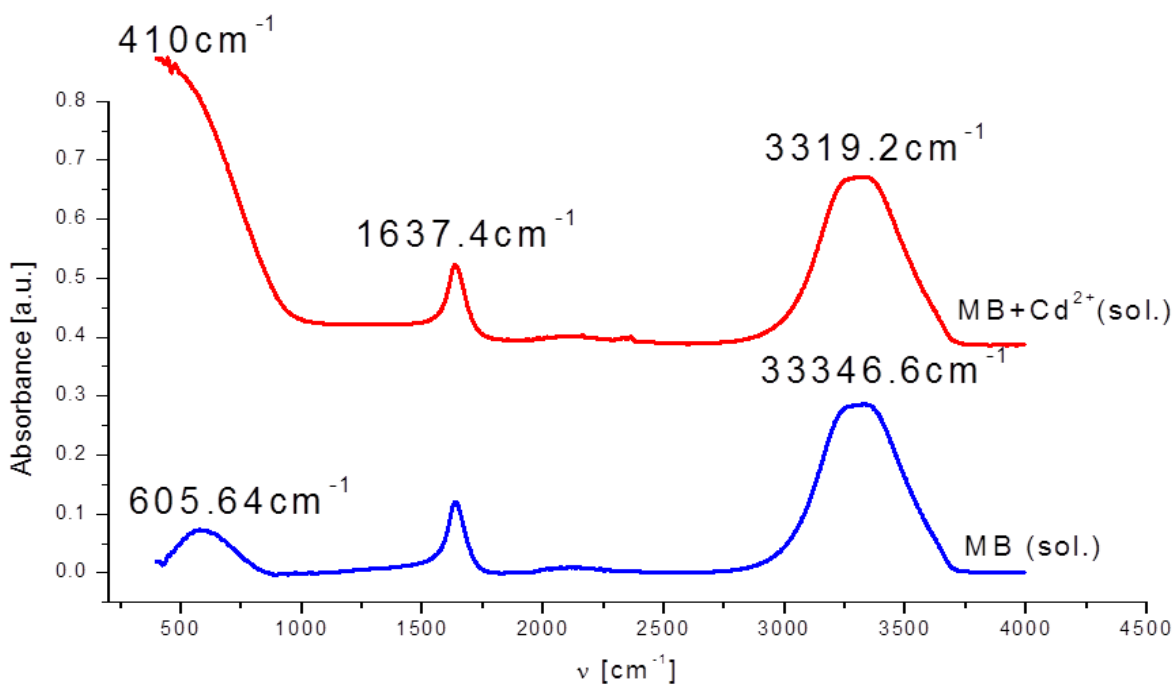

Figure 9: FT-IR spectra of the $M B$ and $M B+C d^{2+}$. 


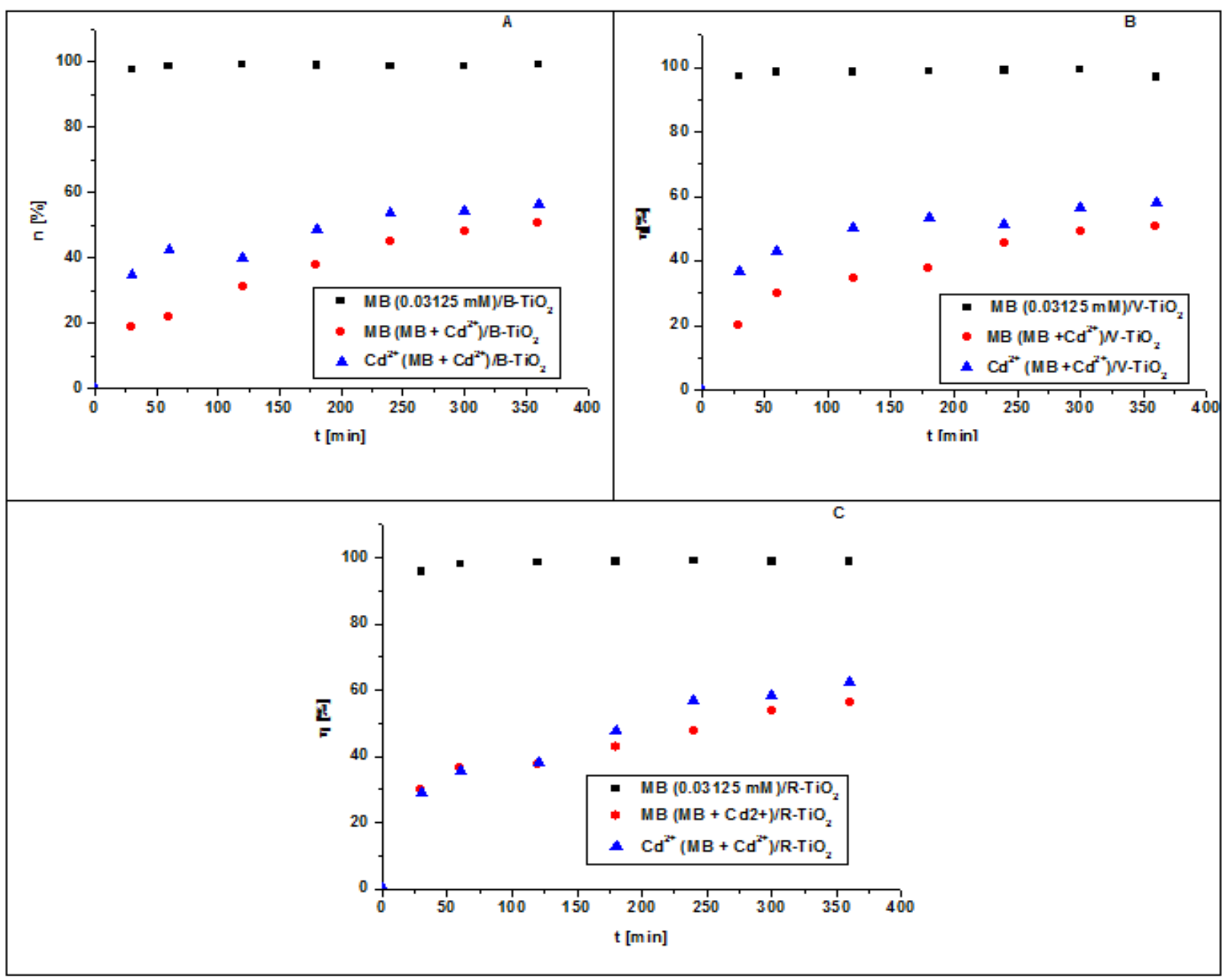

Figure 10: The influence of $\mathrm{H}_{2} \mathrm{O}_{2}+\mathrm{Fe}^{2+}$ in photocatalytic process on: (a) $\mathrm{B}-\mathrm{TiO}_{2^{\prime}}$ (b) $\mathrm{V}-\mathrm{TiO}_{2^{\prime}}$ (c) $\mathrm{R}-\mathrm{TiO}_{2}$.

\begin{tabular}{|c|c|c|c|c|c|c|c|c|c|}
\hline \multirow[b]{2}{*}{$8(\mathrm{~A})$} & \multicolumn{3}{|c|}{ Photocatalysis } & \multicolumn{3}{|c|}{ Photo-Fenton } & \multicolumn{3}{|c|}{ Adsorption } \\
\hline & $\mathbf{R}^{2}$ & $k_{2}(g / m g ~ m i n)$ & $q_{e}(m g / g)$ & $\mathbf{R}^{2}$ & 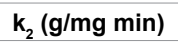 & $q_{e}(m g / g)$ & $\mathbf{R}^{2}$ & $k_{2}$ (g/mg min) & $q_{e}(m g / g)$ \\
\hline B-TiO ${ }_{2}$ & 0.993 & 0.014 & 6.146 & 0.977 & 0.007 & 8.137 & 0.974 & 0.021 & 1.172 \\
\hline $\mathrm{V}-\mathrm{TiO}_{2}$ & 0.985 & 0.027 & 6.845 & 0.997 & 0.021 & 7.704 & 0.971 & 0.019 & 1.337 \\
\hline $\mathrm{R}-\mathrm{TiO}_{2}$ & 0.97 & 0.017 & 5.583 & 0.986 & 0.005 & 7.886 & 0.955 & 0.024 & 1.071 \\
\hline \multicolumn{10}{|l|}{$8(B)$} \\
\hline $\mathrm{B}-\mathrm{TiO}_{2}$ & 0.999 & - & 0.874 & 1 & 4.431 & 0.929 & 0.999 & 0.449 & 0.877 \\
\hline $\mathrm{V}-\mathrm{TiO}_{2}$ & 0.999 & - & 0.914 & 0.999 & - & 0.922 & 0.999 & 0.218 & 916 \\
\hline $\mathrm{R}-\mathrm{TiO}_{2}$ & 0.998 & 0.239 & 0.913 & 0.999 & 0.864 & 0.926 & 0.999 & 0.439 & 0.897 \\
\hline \multicolumn{10}{|l|}{$8(C)$} \\
\hline $\mathrm{B}-\mathrm{TiO}_{2}$ & 0.965 & 0.069 & 0.372 & 0.861 & 0.018 & 0.668 & 0.955 & 0.395 & 0.325 \\
\hline $\mathrm{V}-\mathrm{TiO}_{2}$ & 0.953 & 0.059 & 0.369 & 0.936 & 0.031 & 0.5929 & 0.936 & 1.56 & 0.314 \\
\hline $\mathrm{R}-\mathrm{TiO}_{2}$ & 0.98 & 0.078 & 0.361 & 0.945 & 0.007 & 2.851 & 0.962 & 0.225 & 0.318 \\
\hline
\end{tabular}

Table 8: Kinetic parameters of pollutants $\left((A): \mathrm{Cd}^{2+}\left(M B+C d^{2+}\right)\right.$; $\left.(B): M B ;(C): M B\left(M B+C d^{2+}\right)\right)$ removal in photocatalysis; photo Fenton processes and adsorption.

kinetic parameters were calculated and are presented in Table 8 (A, B and C).

These data show that in the system containing multi pollutant here is the high cadmium mobility comparative to methylene blue, in adsorption and photodegradation process. This is the confirmation that cadmium is firstly adsorbed on the substrate and then methylene blue. The adsorption process of multi pollutant in the experimental condition is significantly controlled by cadmium mobility. 
Citation: Guillaume PLA, Chelaru AM, Visa M, Lassiné O (2018) “Titanium Oxide-Clay” as Adsorbent and Photocatalysts for Wastewater Treatment. J Membra Sci Technol 8: 176. doi:10.4172/2155-9589.1000176

In photo-Fenton process, the $\mathrm{Cd}^{2+}$ cations removal rate is higher than photocatalysis process; the same applied to other pollutants, namely $\mathrm{MB}$. The $\mathrm{HO}^{-}$ions (chemo) sorbs and negatively charge $\mathrm{TiO}_{2}$, resulting in an activated surface with increased affinity for $\mathrm{Cd}^{2+}[26,27]$.

\section{Conclusion}

A new substrate was obtained in a hydrothermal process, starting with clay from regions of north of Ivory Coast, namely: Katiola and Fronan and $\mathrm{TiO}_{2}$ Degussa P25 and was tested for simultaneous removal of heavy metal cations and dyes, in a single step process, involving adsorption and photocatalysis.

The clay- $\mathrm{TiO}_{2}$ structural and morphology analysis showed that the substrate has a high crystallinity degree and surface, homogeneity and good roughness for adsorption of $\mathrm{MB}$ and of cadmium cations.

The adsorption efficiency of $\mathrm{MB}(0.03125 \mathrm{mM})$ and $\mathrm{Cd}^{2+}(0.01 \mathrm{~N})$ on $\mathrm{R}-\mathrm{TiO}_{2}$ is $91.19 \%$ and $27.05 \%$, respectively. For photocatalysis, efficiency increase for the same pollutants on $\mathrm{R}-\mathrm{TiO}_{2}$ from $91.19 \%$ to $97.48 \%$ and from $27.05 \%$ to $44.37 \%$. For photo-Fenton process, there are increases the efficiency of organic pollutants degradation and heavy metal removal than photocatalysis process. The High photo-Fenton efficiency resulted of hydroxides radicals produced in the medium by mixture of $\mathrm{H}_{2} \mathrm{O}_{2}$ and $\mathrm{Fe}^{2+}$.

The kinetic studies show that the substrate has a good adsorption capacity and fast adsorption processes which is, mainly based on the electrostatic attractions between the substrate and the pollutant species. The new substrate has the grains in the micrometric range, representing thus a promising alternative to Degussa P25 slurries. This is a simpler and cost-effective method to recuperate/recycle the substrate in industrial wastewater treatment processes. High adsorption efficiencies are registered for $\mathrm{MB}$, and simultaneous removal of the $\mathrm{MB}$ and $\mathrm{Cd}^{2+}$ is possible, on all materials $\mathrm{B}-\mathrm{TiO}_{2}, \mathrm{~V}-\mathrm{TiO}_{2}, \mathrm{R}-\mathrm{TiO}_{2}$.

\section{Acknowledgments}

This work was financed by Agence Universitaire de la Francophonie (Eugen lonescu scholarship).

\section{References}

1. Global environment outlook 3 (2002) UNEP Nairobi, Kenya

2. Shi D, Ni M, Zeng J, Ye J, Ni P, et al. (2015) Simultaneous detection and removal of metal ions based on a chemosensor composed of a rhodamine derivative and cyclodextrin-modified magnetic nanoparticles. $\mathrm{J}$ of Materials Science 50: 168-175

3. Ge F, Ye H, Li MM, Zhao BX (2012) Efficient removal of cationic dyes from aqueous solution by polymer- modified magnetic nanoparticles. Chem Engin J 198: 11-17.

4. Tunay O, Kabdasli, Eremektar G, Orhon D (1996) Color removal from textile wastewaters. J Water Sci Technol 34: 9-16.

5. Mäusezahl D, Christen A, Pacheco GD, Tellez FA, Iriarte M (2009) Solar Drinking Water Disinfection (SODIS) to Reduce Childhood Diarrhoea in Rural Bolivia: A Cluster-Randomized,Controlled Trial. Plos Medicine 6.

6. Bahnemann DCJ, Fox MA, Pelizzetti E, Pichat P, Serpone N (1994) Photocatalytic Treatment of Waters. Boca Raton, Lewis Publishers.

7. Melián JAH, Rodríguez JMD, Suárez AV, Rendón ET, Do Campo CV, et al (2000) The Photocatalytic disinfection of urban waste waters. Chemosphere pp: 41323-41327.
8. Legrini OE, Braun EM (1993) Photochemical processes for water-treatment Chemical Reviews 93: 671-698.

9. Rincón AG, Pulgarin C, Adler N, Peringer P (2001) Interaction between E. coli inactivation and DBP-precursors - dihydroxybenzene isomers - in the photocatalytic process of drinking-water disinfection with TiO2. Journal of Photochemistry and Photobiology A: Chemistry139: 233-241.

10. Neumann MG, Gessner F, Schmitt CC, Satori R (2002) Influence of the layer charge and clay particle size on the interaction between the cationic dye methylene blue and clay in aqueous suspension. J Colloid Interface Sci 225 : 254-259.

11. Visa M, Bogatu C, Duta A (2015) Tungsten oxide - Fly ash oxide composites in adsorption and photocatalysis. J of Hazard Mater 289: 244-256.

12. Andronic L, Andrasi D, Enesca A, Visa M, Duta A (2011) The influence of titanium dioxide phase composition on dyes phtocatalysis. J Sol-Gel Sci Technol 58: 201-208

13. Visa M (2012) Tailoring fly ash activated with bentonite as adsorbent for complex wastewater treatment. J Appl Surf Sci 263: 753-762.

14. Šljivić M, Smičiklas I, Pejanović S, Plećaš I (2009) Comparative study of $\mathrm{Cu}^{2+}$ adsorption on a zeolite, clay and a diatomite from Serbia. J of Applied Clay Science 43: 33-40

15. Khorsand Zak A, Majid WHA, Abrishami ME, Yousefi R, Parvizi R (2012) Synthesis, magnetic properties and $\mathrm{X}$-ray analysis of $\mathrm{Zn} 0.97 \mathrm{X} 0.030$ nanoparticles $(X=\mathrm{Mn} \mathrm{Ni}$, and $\mathrm{Co})$ using Scherer and size-strain plot methods. Solid State Sci. 14: 488-494.

16. Duta A, Visa M (2015) Simultaneous removal of two industrial dyes by adsorption and photocatalysis on a fly-ash- $\mathrm{TiO}_{2}$ composite. $\mathrm{J}$ of Photochemistry and Photobiology A-Chemistry pp: 30621-30630

17. Devi LG, Kumar SG (2012) Exploring the critical dependence of adsorption of various dyes on the degradation rate using $\mathrm{Ln}^{3+}-\mathrm{TiO}_{2}$ surface under UV/solar light. Appl Surf Sci 261: 137-146.

18. Irawatya W, Soetaredjoa FE, Ayucitraa A (2014) Understanding the relationship between organic structure and mineralization rate of $\mathrm{TiO}_{2}$-mediated. Procedia Chemistry 9: 131-138.

19. Jing L, Li S, Song S, Xue L, Fu H (2008) Investigation on the electron transfer between anatase and rutile in nano-sized $\mathrm{TiO}_{2}$ by means of surface photo voltage technique and its effects on the photocatalytic activity. Solar Energy Mater Solar Cells 92: 1030-1036.

20. Visa M, Bogatu C, Duta A (2010) Simultaneous adsorption of dyes and heavy metals from multicomponent solutions using fly ash. J of Applied Surface Science 256: 5486-5491.

21. Fujishima A, Rao TN, Tryk DA (2000) Titanium dioxide photocatalysis. J Photochem Photobiol C: Photochem Rev 1: 1-21.

22. Song C, Zhang J (2008) Electrocatalytic oxygen reduction reaction in PEM Fuel cells electroctalaysts and catalyst layers. Fundamental and Applications, Springer pp: 89-133.

23. Fenton HJH (1894) Oxidation of tartaric acid in presence of iron. J Chem Soc Trans 65: 899-910.

24. Amilcar Machulek Jr., Frank H. Quina, Fabio Gozzi, Volnir O. Silva, Leid C. Friedrich, et al. Fundamental Mechanistic Studies of the Photo-Fenton Reaction for the Degradation of Organic Pollutants Brazil

25. Ho YS, McKay G (1999) Pseudo-second order model for sorption processes Process Biochem pp: 3451-3465.

26. Visa M, Duta A (2008) Advanced $\mathrm{Cd}^{2+}$ Removal on Dispersed $\mathrm{TiO}_{2}-$ Fly Ash Environmental Engineering and Management J 7: 373-378.

27. Visa M, Duta A (2010) Adsorption Behavior Of Cadmium And Copper Compounds On A Mixture FA: $\mathrm{TiO}_{2}$. Rev Roum Chim 55: 167-173. 